\title{
Signal Timing Optimization Model for Intersections in Traffic Incidents
}

\author{
Jiawen Wang (D, Jiayu Hang, and Xizhao Zhou \\ Business School, University of Shanghai for Science and Technology, Shanghai 200093, China \\ Correspondence should be addressed to Jiawen Wang; wangjw@usst.edu.cn
}

Received 20 November 2019; Revised 15 May 2020; Accepted 2 September 2020; Published 14 September 2020

Academic Editor: Giulio E. Cantarella

Copyright (c) 2020 Jiawen Wang et al. This is an open access article distributed under the Creative Commons Attribution License, which permits unrestricted use, distribution, and reproduction in any medium, provided the original work is properly cited.

\begin{abstract}
The intersection control and management can alleviate the traffic congestion caused by traffic incidents. Therefore, it becomes essential to develop a signal optimization method for intersections influenced by traffic incidents, which will be beneficial to prevent congestion spreading. In this paper, the proposed model is capable of maximizing the intersection throughput by comprehensively considering the queue length as the penalty value. The headway of leaving vehicles is assumed to follow the Cowan's M3 headway distribution, where formulas for queue length can be derived based on gap acceptance theory. To satisfy the conditions for efficiently identifying feasible solutions in a short time, a heuristic algorithm (simulated annealing algorithm) is employed to solve the model. The numerical results can validate that the proposed method can solve the problem more efficiently and alleviate the intersection congestion caused by the incidents more desirably. When the incident occurs away from the intersection stop line, the impacts on intersection throughput will be gradually weakened. The proposed method is capable of improving the signalized intersection throughput while preventing the congestion from spreading to the upstream intersection.
\end{abstract}

\section{Introduction}

Incidents refer to all the events such as accidents, dropped cargo, road construction, illegal temporary parking, and train passing, which affect (or may affect) the capacity of the road and hinder smooth traffic flow. If incidents occur at an intersection, vehicle queuing will occur, which will indirectly result in the time resources waste [1]. Secondary accidents may also occur in accidental areas [2]. In urban road network, the intersections are highly correlated [3]. When vehicles queue up to the upstream intersection, the congestion will inevitably diffuse to adjacent intersections and worsen the situation [4]. The network gridlock may arise in severe cases [1]. There are two main ways of dealing the urban road network problems in emergency incidents. (i) The traffic capacity can be increased to mitigate the impact of incidents, e.g., road network infrastructure construction, roads widening, subway building. (ii) More traffic incident management methods and integrated control and management of urban traffic operations and facilities can be applied to fully leverage the existing road network resources and obtain optimal traffic operation [5], e.g., traffic priority management $[6,7]$, intelligent traffic management system $[8,9]$, and abnormal traffic management $[10,11]$. It is apparent that more urban land resources will be occupied in the first way, which requires a long time to work. Due to the stochasticity and uncertainty of traffic incidents, it will result in inefficient traffic management by blindly increasing traffic capacity through infrastructure transformation $[12,13]$. Therefore, it becomes important to utilize advanced traffic management methods to solve the urban road network problems caused by traffic incidents. The urban traffic control system is an important part of the advanced traffic management system (ATMS), where seeking a reasonable signal control strategy for intersections in incidents has become the research focus in ATMS.

The research on signal control at urban intersections in incidents can be mainly summarized into two aspects. The first one focuses on signal priority for emergency vehicles, and the other one concentrates on the traffic congestion mitigation in traffic incident area. 
Various literatures have been published on emergency vehicle (EV) preemption. Two new control strategies for emergency vehicle signal preemption (EVSP) is proposed by Qin et al. [14-16]. In the first strategy, a real-time control scheme is developed, which can enable signal transitioning from normal operation to EVSP. The other control strategy is implemented with an optimal control algorithm, which can be used for transitioning the signal from the EVSP back to normal operation. A two-phase algorithm is developed to solve the optimal control model, which consists of a relaxation method and a stepwise search strategy [14-16]. An emergency vehicle signal coordination (EVSC) approach is proposed, which can provide "green wave" for EVs in signal coordination setting [17-19]. The timed Petri nets (TPNs) is employed by Huang et al. to model preemption of emergency vehicle systems for the first time. The proposed approach can clearly illustrate the traffic light behaviors in terms of conditions and events, where the preemption of phases is being changed $[20,21]$.

Some researchers focus on the signal timing optimization for traffic congestion mitigation. Based on the graph theory, system partitioning and the route guidance are proposed by Xie. for developing traffic congestion control model, where the Kruskal test was utilized to solve the formulated model [22]. To account for traffic incidents at different locations of road network, He et al. has developed different congestion evacuation schemes for signalized intersections with a cell transmission model [23]. Based on the vehicle information probed by GPS, a global network-level analysis model is proposed for estimating traffic condition in network after the emergency by matrix factorization and clustering methods $[24,25]$.

In all the previous researches, it has been shown that traffic signal optimization strategies for emergencies are helpful in mitigating the traffic congestion. However, the stochasticities of incidents such as the location of incident in the intersection, have not been fully considered in some of the research.

It should be noted that the existing research is capable of improving the intersection management in certain conditions. However, where the stochastic traffic incidents happen in the intersection area, i.e., departure road or approach road, will lead to different the congestion area, which may influence the performance of the signal timing optimization. As a result, it is essential to propose a signal timing optimization method considering the location of incident in the intersection. In this paper, we will fill this gap by comprehensively considering the stochasticity of the location of incident in the intersection, where the model for signal timing optimization will also be established in traffic incidents.

This paper proposes a signal timing optimization model to maximize intersection throughput considering the restriction of queue length aroused by traffic incidents. The simulated annealing algorithm is used to solve the constructed signal timing optimization model. The computation time of the proposed technique is sufficiently short, where finding feasible solutions becomes possible. The contribution of this paper can be summarized in the following two perspectives: (i) a signal timing optimization method is proposed for two-phase signal control intersection, where the queue length restriction caused by incidents is considered and (ii) the variation of throughput influenced by various factors (i.e., saturation, distance between incident and stop line) is validated by numerical simulation. Compared with original fixed signal timing plan, numerical results have shown that the proposed signal control method can improve the throughput of the intersection effectively. Especially in the case of medium traffic flow saturation, the calculated signal timing plan is able to improve intersection throughput whether incident occurs at the approach road or at departure road. In contrast, the impacts on throughput can be gradually weakened when the incident occurs away from the intersection stop line.

The remainder of this paper is organized as follows. In Section 2, an introduction on signal timing optimization model for intersection influenced by traffic incidents is given. In Section 3, the queue length formulas are derived and a new model is proposed. The solving procedures of the proposed model are based on simulated annealing algorithm as introduced in Section 4. In Section 5, numerical experiments are presented under varying traffic demand. In Section 6, the conclusion and discussion on future research directions are presented.

\section{Problem Description}

In urban road network, traffic incidents have impact on operational performance of signal control intersections. As shown in area Figures 1(a) and 1(b), traffic incidents can randomly occur at different locations in the intersection. Therefore, it is required to use corresponding measures to maintain the intersection operation. The vehicle firstly starts to queue at departure No. 4 when an incident occurs in area (a). Some vehicles will queue up at approach No. 2 and No. 3 for preventing the formation of export blockage. To prevent approach spillback from occurring, it becomes necessary to control the queue length at approach No. 2 and No. 3. The queue length at the incident area is also required to be controlled when an incident occurs in area (b). In this paper, a two-phase signal timing optimization method is proposed by considering the locations where incidents occur. In this manner, the intersection throughput can be maximized.

If incident occurs on approach road, as in area (b), the arriving vehicles leave the incident affected link by crossing the gap of traffic flow on the departure road in the other direction. Hence, the headway distribution on departure road has a more significant impact on the intersection throughput compared with that on approach road has. Therefore, the flow on approach road is simplified to be a constant flow, and headway distribution on departure road is assumed to obey the Cowan's M3 headway distribution.

Commonly used distribution functions include negative exponential distribution [26], shifted negative exponential distribution [27], Erlang distribution [28], Cowan's M3 headway distribution [29], etc. Yao et al. used trajectory data for cycle-based flow estimation through a generic hybrid 


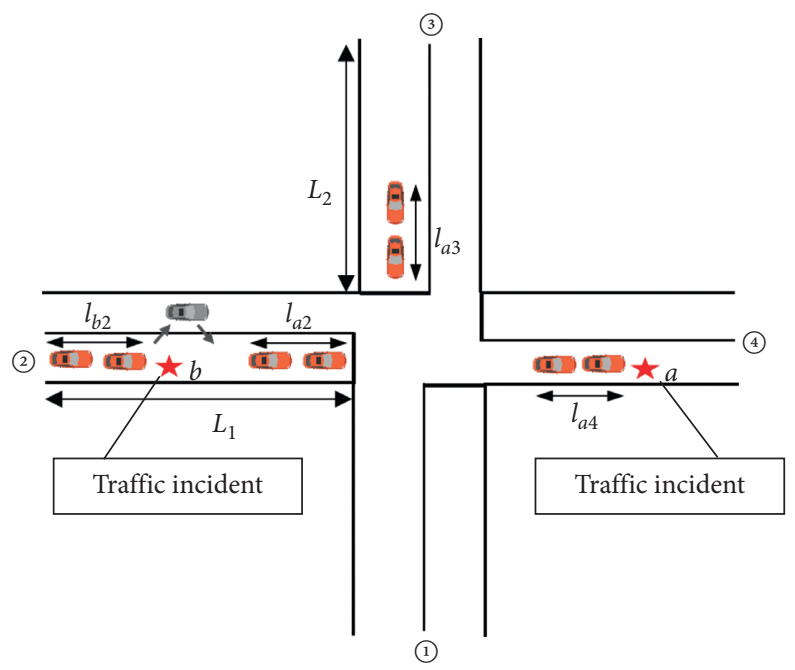

Figure 1: An intersection layout influenced by traffic incidents.

method that combined a probabilistic model and shockwave theory. In their method, within each cycle, the volume of nonstopped vehicles is modeled as a parameter estimation problem of a time-dependent constrained Poisson distribution, where the time headway correspondingly obeys a Cowan's M3 headway distribution [30]. Li et al. obtain the time headway data of different road section and different lanes on the downstream section of signalized intersection. They adopt the chi-squared test to analyze the time headway distribution and obtain the characters of different road section and different lanes in the peak period and the normal period. The results indicate that with the time elapsing from the peak to normal period and the vehicles moving from the intersection to downstream section, the time headway distribution on the lanes is transformed between the shifted negative exponential distribution and Cowan's M3 headway distribution [31].

Based on the research above, it can be concluded that though the application of Cowan's M3 headway distribution in signalized intersections is defective, it is feasible to assume that the traffic flow at the departure road obeys Cowan's M3 headway distribution in certain circumstances.

The actual headway data are utilized to verify the distribution of headways of the departure road. The fitted data are shown in Table 1, which is assumed to obey Cowan's M3 headway distribution. The chi-squared test method is used. $\alpha$ is $0.05 . \chi^{2}$ is 10.78. After checking the table, $\chi_{0.05}^{2}$ is 11.070, which is more than $\chi^{2}$. It is verified that the hypothesis is acceptable. Hence, in this paper, the distribution of headway on departure road at the intersection is assumed to obey Cowan's M3 headway distribution.

Vehicles departs the incident section from the exit road (departure No. 2) if the incident occurs in area (b). The vehicles can successfully cross the counterflow only when the headway is more than crossable gap. The queue length can be then used as a penalty value in the objective function if it becomes higher than a certain value. The flowchart of signal timing optimization affected by traffic incidents can be given in Figure 2.

\section{Signal Timing Optimization Model}

3.1. Parameter Description. To facilitate the model formulation, the key notations used hereafter are summarized in Abbreviations.

3.2. Queue Length in Traffic Incidents. In this section, queue length calculation in traffic incidents will be derived systematically. According to the location of traffic incident, the queue length calculation can be divided into two cases. Figure 3 illustrates the queue length caused by traffic incidents in departure road and in approach road. In this paper, the signal timing optimization of the two-phase intersection is investigated for traffic incidents. Right-turn traffic flow is unbounded at the intersection. Therefore, the queue length of right-turn traffic flow is not considered.

3.2.1. Traffic Incident Occurs on Departure Road. As shown in Figure 3(a), the traffic capacity of signal control intersection is reduced when the incident occurs at departure No. 4. The straight traffic flow of approach No. 2 and left-turn traffic flow of approach No. 3 appear to be queuing. In this paper, the influence coefficient of traffic incidents on the saturation flow rate $\beta$ is introduced, which is used to reflect the traffic incident effects on intersection throughput. Subsequently, the queue length of approach No. 2 and approach No. 3 can be calculated by

$$
\begin{aligned}
& l_{a 2}=q_{2 s} T-\left(\beta s_{2} X_{1}+\frac{L_{0}}{l_{c}}\right), \\
& l_{a 3}=q_{3 l} T-\left(\beta s_{3} X_{2}+\frac{L_{0}}{l_{c}}\right) .
\end{aligned}
$$

3.2.2. Traffic Incident Occurs on Approach Road. As shown in Figure 3(b), the traffic capacity of signal control intersection is reduced when the incident occurs at the approach 
TABLE 1: Vehicles leaving the departure road.

\begin{tabular}{lcc}
\hline Time headway $(s)$ & Frequency of cumulative observation & Frequency of theoretical observation \\
\hline$(0,3]$ & 70 & 71.4015 \\
$(3,6]$ & 62 & 50.1202 \\
$(6,9]$ & 28 & 31.3240 \\
$(9,12]$ & 13 & 19.5570 \\
$(12,15]$ & 6 & 12.2385 \\
$(15,18]$ & 8 & 7.6465 \\
$>18$ & 18 & 12.7305 \\
\hline
\end{tabular}

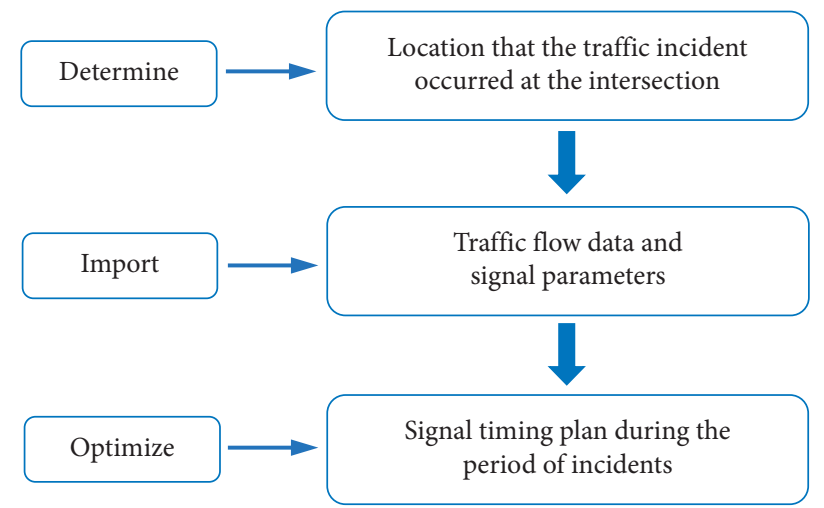

FIgURE 2: The flowchart of signal timing optimization affected by traffic incidents.

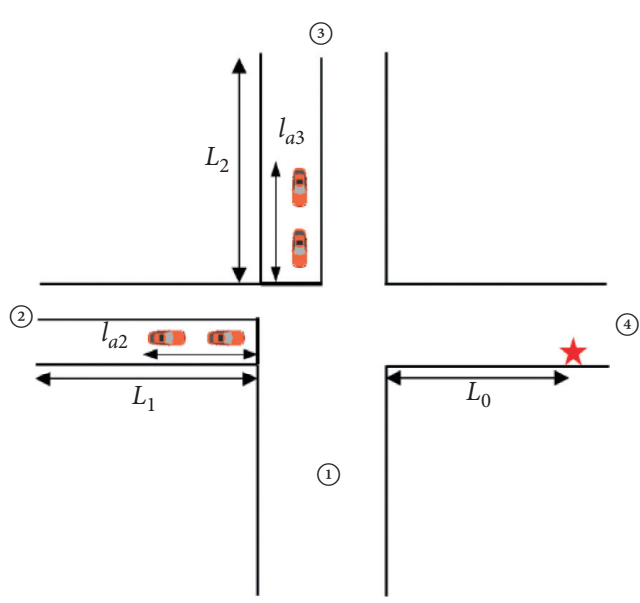

(a)

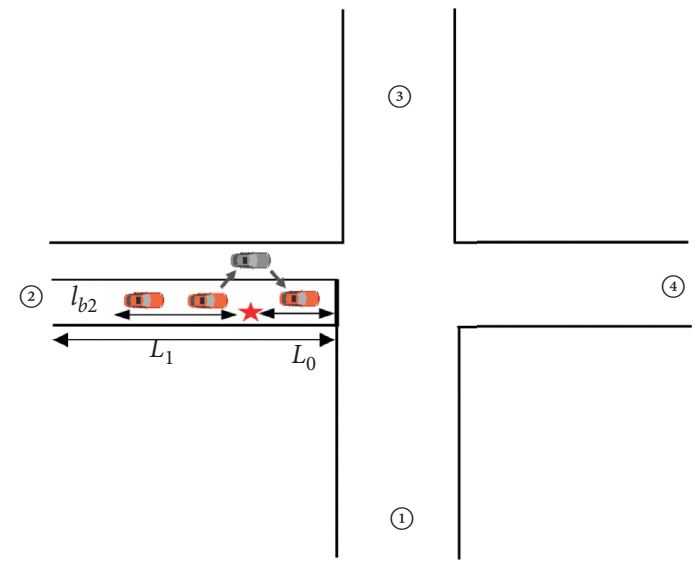

(b)

Figure 3: The queue length when traffic incidents occur in (a) No. 4 departure and (b) No. 2 approach.

No. 2. When vehicles queueing in incident area occurs in the traffic flow of approach No. 2, vehicles need to leave the incident section with the help of exit road (departure No. 2). Based on accepted gap theory, the queue length calculation at incident area is able to be derived on the condition that the traffic flow of the approach No. 4 is controlled by the Cowan's M3 headway distribution. The Cowan's M3 headway distribution reveals that some vehicles drive in a fleet state when traffic is crowded, and some vehicles drive in a free-flow state. The probability density function $f(t)$ and the distribution function $F(t)$ can be given as follows:

$$
\begin{aligned}
& f(t)= \begin{cases}\gamma \lambda e^{-\lambda\left(t-t_{m}\right)}, & t \geq t_{m}, \\
0, & t<t_{m},\end{cases} \\
& F(t)= \begin{cases}1-\gamma e^{-\lambda\left(t-t_{m}\right)}, & t \geq t_{m}, \\
0, & t<t_{m} .\end{cases}
\end{aligned}
$$

The maximum likelihood estimation of $\gamma$ is

$$
\widehat{\gamma}=1-q_{i} t_{m}
$$

The maximum likelihood estimation of $\lambda$ is 


$$
\widehat{\lambda}=\frac{\gamma q_{i}}{1-q_{i} t_{m}}=q_{i}
$$

where $P$ is a random number following the $0-1$ distribution with $P=f(t)$. Subsequently, the inverse function of headway can be derived as

$$
t=t_{m}-\frac{1}{\lambda} \ln \frac{1-P}{\gamma} .
$$

The straight traffic flow of approach No. 4 and left-turn traffic flow of approach No. 1 during one cycle can be given as

$$
\begin{aligned}
& q_{4 s} X_{1}=N_{4}, \\
& q_{1 l} X_{2}=N_{1} .
\end{aligned}
$$

Subsequently, the queue length during one cycle in the influence of traffic incident occurs in approach No. 2 can be derived as

$$
l_{b 2}=q_{2} T-\left(\sum_{k=1}^{N_{4}} \frac{t_{4 k}-t_{c}}{t_{f}}+\sum_{k=1}^{N_{1}} \frac{t_{1 k}-t_{c}}{t_{f}}\right) .
$$

3.2.3. Signal Timing Optimization Model under the Influence of Traffic Incidents. When traffic incident occurs at intersection, the capacity will decline significantly. The incident affected approach road will be crowded and vehicles will appear to queue. The goal of signal timing optimization is to increase the throughput of intersections. When the queue spills back and affects the upstream intersection, the queue length is required to be controlled. Therefore, the objective function will increase by adding the queue length penalty value according to the penalty function theory, where the signal optimization method will be performed in traffic incidents as shown in the following formula:

$$
\begin{aligned}
& \max F\left(X_{j}\right)=\frac{\beta s_{i} X_{j}}{T}-\alpha\left[\varepsilon \max \left(0, \Delta L_{a i}\right)+(1-\varepsilon) \max \left(0, \Delta L_{b i}\right)\right]
\end{aligned}
$$

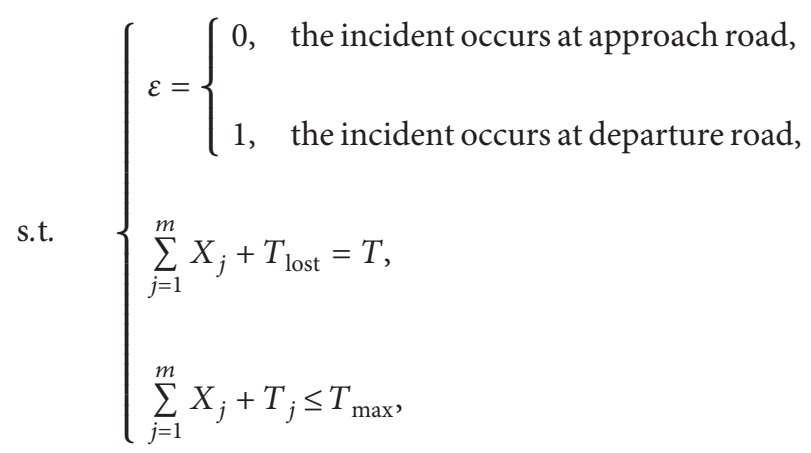

$$
\begin{aligned}
& \Delta L_{a i}=l_{c}\left(l_{a i}-L_{i}\right), \\
& \Delta L_{b i}=l_{c}\left(l_{b i}-L_{i}\right) .
\end{aligned}
$$

\section{Algorithm}

The simulated annealing algorithm is a stochastic optimization method, which is based on the use of Monte Carlo iterative solution [32]. In solving local optimal and global optimal problems, it can exhibit outstanding performance [33]. Essentially, this algorithm learns from the physical annealing process of solids. As the solid temperature decreases, it gradually balances and reaches to the ground state, where convergence to the global optimal solution can be obtained. In particular, the solution of simulated annealing algorithm is independent of the initial solution. In fact, it can accept the bad solution with a certain probability, which will be used to eliminate local optimal solutions and achieve the global optimal solution ultimately [34]. The determination of initial temperature and temperature cooling rate are the key techniques in applying the algorithm. If the initial temperature is high enough, the global optimal solution can be obtained. However, the computational time of the algorithm increases with the increase in the initial temperature. Therefore, the initial temperature should be selected appropriately. Similarly, the temperature will decrease slowly when the temperature cooling rate is very low, where the probability of convergence to the optimal solution can be increased. Some researchers have been conducted on the key parameters in the simulated annealing algorithm [35]. The experimental results demonstrate that it can obtain the best algorithm solution when the temperature cooling rate is 0.95 and the final temperature is 0.1 [36].

The main procedures of the simulated annealing algorithm can be summarized as follows. The flow chart can be shown in Figure 4. 


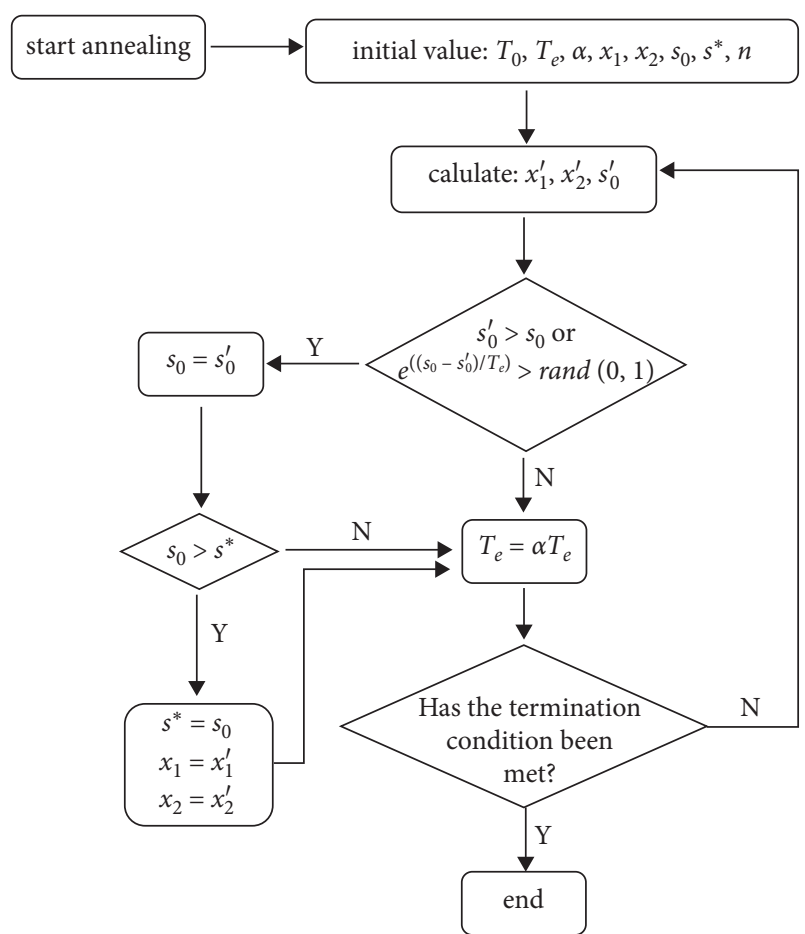

FIgURE 4: The signal timing optimization flow chart with simulated annealing algorithm.

Step 1. Initialization: the initial temperature $T_{0}$ is set to be large enough. Let $T_{e}$ be $T_{0}$. To determine the initial solution, the signal timing can be calculated by the Webster method in this paper. The initial signal timing is $x_{1}, x_{2}$. By calculating the initial objective function value $s_{0}$, we set the global optimal solution $s^{*}$ to be equal to $s_{0}$. Determine the number of iterations $n$, the temperature cooling rate $\alpha$.

Step 2. Repeat the third step to the sixth step.

Step 3. Perform a random disturbance on the initial signal timing. The new signal timing $x_{1}^{\prime}, x_{2}^{\prime}$ will be calculated, and new objective function $s_{0}^{\prime}$ can be obtained.

Step 4. If $s_{0}^{\prime}$ is more than $s_{0}$, $s_{0}$ will be set to be $s_{0}^{\prime}$. If not, the acceptance probability $e^{\left(s_{0}-s_{0}^{\prime}\right)} / T$ will be calculated. If $e^{\left(s_{0}-s_{0}^{\prime}\right)} / T$ is larger than a random number between 0 and $1, s_{0}$ will be set to be $s_{0}^{\prime}$.

Step 5. If the new $s_{0}$ is larger than $s^{*}, s^{*}$ will be set to be $s_{0}$ and the new signal timing $x_{1}^{\prime}, x_{2}^{\prime}$ will be obtained. If not, the global optimal solution $s^{*}$ will remain to be unchanged.

Step 6. Calculate the new temperature $T_{e} ; T_{e}$ is equal to $\alpha T_{e}$.

Step 7. If the termination condition is satisfied, $s^{*}$ will be used as the global optimal solution, and the optimal signal timing is $x_{1}^{\prime}, x_{2}^{\prime}$. If the number of iterations is met, the iterations in the algorithm terminate.

\section{Numerical Study of Signal Timing Optimization}

In this section, the numerical study will be given, where various factors have been considered to investigate their effects on signal timing optimization. In the following, a brief summary of the assumptions is presented, which have been made before performing the simulation.

(1) If traffic incidents occur on the approach/departure road, the vehicles will not queue at intersections.

(2) The saturation flow rate of the through lane in incidents is assumed to be constant.

(3) If traffic incidents occur at the intersection, the proposed method will be performed to optimize the signal timing. If there is no traffic incident, the original control strategy is implemented at the intersection.

To illustrate the applicability of the proposed model, in this section, an optimization is proposed for the signal timing plan for a two-phase signal control intersection, which is affected by an incident. The pretimed signal control strategy is implemented in the intersection when there are no traffic incidents.

It is assumed that the traffic incident occurs at the intersection in the simulation. Intersection throughput can be calculated by the parking line method under the pretimed signal control [37]. Subsequently, the proposed model can be used to optimize the signal timing of the traffic incident influenced intersection, where the intersection throughput can be calculated. Finally, the intersection throughput can be compared before and after the optimization. The key parameters are summarized in Table 2.

In Figure 5, intersection throughput before and after the signal optimization is compared in traffic incidents with saturation from 0.4 to 0.9. As shown in Figure 5(a), when the incident occurs at the approach road, the proposed simulated annealing based signal control method is capable of effectively improving the throughput of the intersection, compared with the original fixed signal timing plan. When the saturation degree is between 0.4 and 0.5 , the optimization is not effective. When the saturation increases, the intersection throughput can be significantly improved. However, the signal optimization effects are weakened, when the traffic flow is close to saturation. A possible explanation might be that the robustness of signal control for the intersection with high saturated traffic flow becomes poor. When traffic incident occurs, the operation of the intersection will be significantly affected, where the intersection throughput cannot be improved through signal timing optimization. As shown in Figure 5(b), the results of signal timing optimization when the incident occurs at the departure road are similar to those when the incident occurs at the approach road. As shown in Figure 5, it can be concluded by comparing the red box area in Figure 5(a) with in Figure 5(b) that the proposed method can achieve better signal timing optimization when the traffic incident occurs at the departure road of the intersection. According to the above analysis, it can be concluded that the proposed approach can be useful in improving the intersection operational efficiency when traffic incidents occur.

In Figure 6, the variation of throughput is illustrated when incidents occur at different locations in approach road of the intersection. As can be seen from Figure 6, the impacts 
TABLE 2: The key parameters.

\begin{tabular}{lcc}
\hline Name of parameters & Value & \\
\hline$t_{c}$ & 5.36 & Unit \\
$t_{f}$ & 2.86 & $\mathrm{~s}$ \\
$t_{m}$ & 2 & $\mathrm{~s}$ \\
$l_{c}$ & 7 & $\mathrm{~s}$ \\
$s_{i}$ & 1800 & $\mathrm{~m}$ \\
$\beta$ & 0.8 & $(\mathrm{pcu}) / \mathrm{h}$ \\
\hline
\end{tabular}

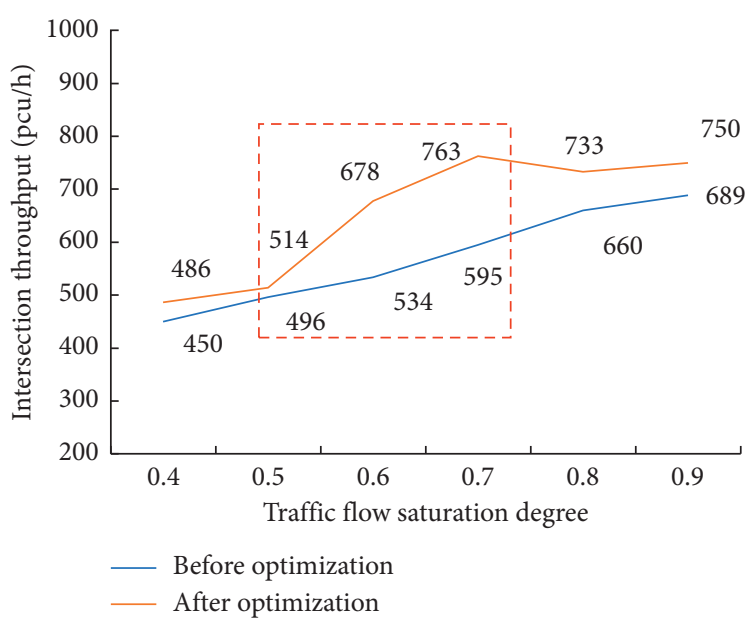

(a)

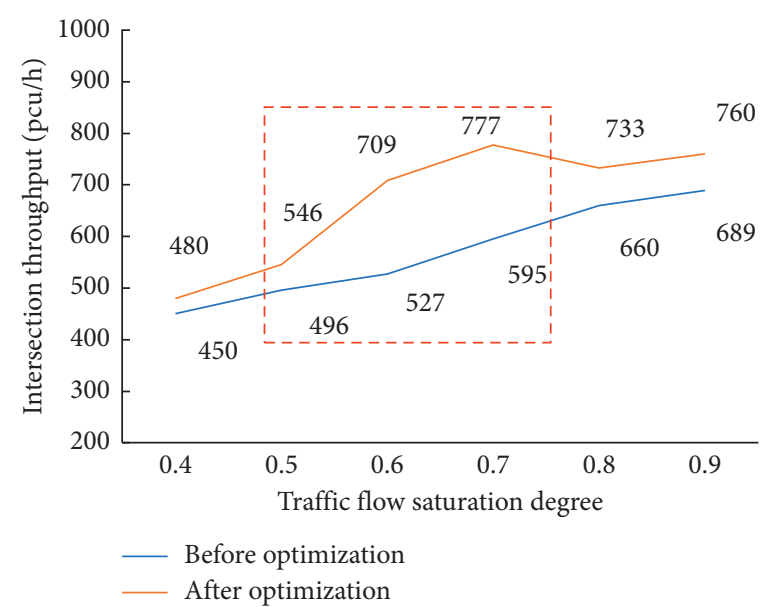

(b)

FIGURE 5: Intersection throughput before and after signal optimization in traffic incident. The incident occurs at (a) the approach road and (b) the departure road.

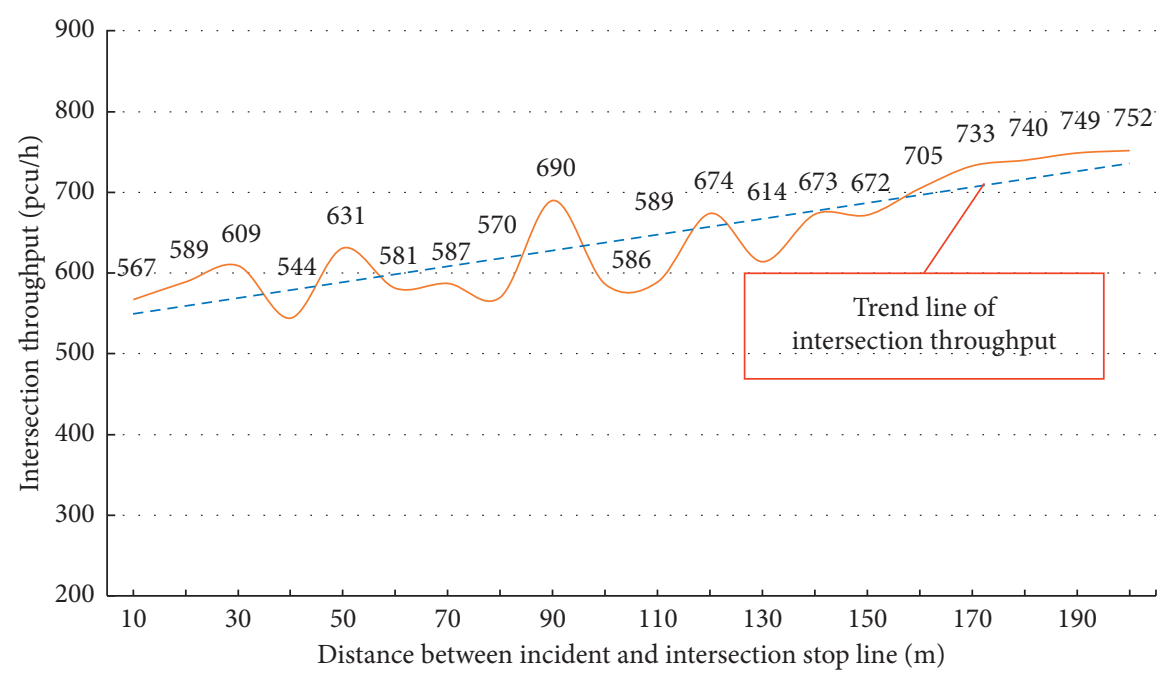

Figure 6: Impacts of incident locations on intersection throughput.

on throughput are gradually weakened when the incident occurs away from the intersection stop line. In other words, when the traffic incident happens close to the intersection, it will have serious impact on the intersection operation, where improving the intersection throughput through signal timing optimization becomes more challenging.

\section{Conclusions}

In this paper, a signal timing optimization method for intersections is proposed, which can consider the influence of traffic incidents. In particular, the gap acceptance theory is introduced in the proposed method. A procedure is proposed for calculating the signal timing plan by considering 
the incident. In other words, the performed approach can take different measures to perform the optimization, based on location of the incident (in the approach road or departure road). In this paper, the simulated annealing algorithm is employed to obtain a signal control scheme by maximizing the intersection throughput. Moreover, the numerical study is performed by taking into various factors (i.e., saturation and distance between incident and stop line). It can be seen from the numerical results that the proposed signal timing plan can improve the intersection throughput effectively with traffic incident.

In summary, it is critical to improve the intersection throughput in traffic incidents, which can improve the operational efficiency of urban transport systems. In this paper, however, the signal timing optimization for twophase intersection is only considered. Therefore, it is potentially important to investigate signal timing optimization of intersections for different control types.

\section{Notations}

$$
\begin{aligned}
& T^{T:} \\
& T_{\max }: \\
& X_{j}: \\
& T_{j}: \\
& T_{\text {lost }}: \\
& t_{c}: \\
& t_{f}: \\
& t_{m}: \\
& t_{i k}: \\
& L_{i}: \\
& l_{c}: \\
& l_{a i}: \\
& l_{b i}: \\
& L_{0}: \\
& \Delta L_{a i}:
\end{aligned}
$$

Cycle length, $s$

Maximum cycle length, $s$

Green time of $j$-th phase, $s ; j=1,2$

Lost time during $j$-th phase, $s ; j=1,2$

Total lost time, $s$
The critical gap of vehicle crossing the counterflow, s

The headway of vehicle crossing the counter-

\section{flow, s}

Minimum headway, $s$

Headway of $k$ vehicle at $i$-th approach, $s$

The maximum queue length of $i$-th approach, $\mathrm{m}$ Space headway, $\mathrm{m}$

Traffic incident occurs on the departure road, the queue length of the $i$-th approach, $\mathrm{m}$

Traffic incident occurs on the approach road, the queue length of $i$-th approach, $\mathrm{m}$

The distance between traffic incident and intersection, $\mathrm{m}$

$\Delta L_{i}=l_{a i}-L_{i}$, the difference between queue length and the maximum queue length of $i$ approach in the influence of traffic incidents occurring at departure road, $m$

$\Delta L_{b i}: \quad \Delta L_{b i}=\left(l_{b i}+L_{0}\right)-L_{i}$, the difference between queue length, $L_{0}$, and the maximum queue length of $i$-th approach in the influence of traffic incidents occurring at approach road, $\mathrm{m}$

$q_{i s}, q_{i l}, q_{i r}$ : The through, left-turn, right-turn traffic flows of $i$ approach

$s_{i}$ : $\quad$ The saturation flow of $i$-th approach, $\mathrm{pcu} / \mathrm{h}$

$v / c: \quad$ Ratio of traffic volume to capacity

$\alpha: \quad$ Penalty factor

$\beta$ : $\quad$ Influence coefficient of traffic incidents on saturated flow rate

$\varepsilon$ : The location of traffic incidents at intersection, $\varepsilon=0,1$. If $\varepsilon$ is 0 , the incident occurs at approach road; if $\varepsilon$ is 1 , the incident occurs at departure road
$F(x): \quad$ The throughput of $i$-th approach in the influence of traffic incidents, $\mathrm{pcu} / \mathrm{h}$

$f(t): \quad$ Probability density function of headway

$F(t): \quad$ Distribution function of headway

$\gamma: \quad$ Free-flow ratio with headway greater than $t_{m}$

$\lambda: \quad$ Attenuation parameters

$P: \quad$ Random number following the 0-1 distribution

$T_{0}$ : $\quad$ Initial temperature

$T_{e}: \quad$ Particle temperature

$\alpha: \quad$ The temperature cooling rate

$x_{1}, x_{2}: \quad$ The initial signal timing

$s^{*}: \quad$ Global optimal solution

$n$ : $\quad$ Number of iterations

$x_{1}^{\prime}, x_{2}^{\prime}: \quad$ New signal timing

$s_{0}: \quad$ The initial objective function

$s_{0}^{\prime}$ : $\quad$ New objective function.

\section{Data Availability}

All the data used to support the findings of this study are included within the article.

\section{Conflicts of Interest}

The authors declare that they have no conflicts of interest.

\section{Acknowledgments}

This work was financially supported by the Shanghai Sailing Program (Grant no. 19YF1435100).

\section{References}

[1] W.-P. Fei, G.-H. Song, F. Zhang, Y. Gao, and L. Yu, "Practical approach to determining traffic congestion propagation boundary due to traffic incidents," Journal of Central South University, vol. 24, no. 2, pp. 413-422, 2017.

[2] C. Sun, X. Pei, J. Hao, Y. Wang, Z. Zhang, and S. C. Wong, "Role of road network features in the evaluation of incident impacts on urban traffic mobility," Transportation Research Part B: Methodological, vol. 117, pp. 101-116, 2018.

[3] Y. Bie, D. Wang, and X. Qu, "Modelling correlation degree between two adjacent signalised intersections for dynamic subarea partition," IET Intelligent Transport Systems, vol. 7, no. 1, pp. 28-35, 2013.

[4] H. Xu, J. Chen, and J. Xu, "Integration of model-based signal control and queue spillover control for urban oversaturated signalized intersection," Mathematical Problems in Engineering, vol. 2019, Article ID 3149275, 2019.

[5] F. Ahmed and Y. E. Hawas, "An integrated real-time traffic signal system for transit signal priority, incident detection and congestion management," Transportation Research Part C: Emerging Technologies, vol. 60, pp. 52-76, 2015.

[6] Q. Lu and K. D. Kim, "A genetic algorithm approach for expedited crossing of emergency vehicles in connected and autonomous intersection traffic," Journal of Advanced Transportation, vol. 2017, Article ID 7318917, 2017.

[7] S. Yagar and B. Han, "A procedure for real-time signal control that considers transit interference and priority," Transportation Research Part B: Methodological, vol. 28, no. 4, pp. 315-331, 1994. 
[8] M. S. Sheikh, J. Liang, and W. S. Wang, "An improved automatic traffic incident detection technique using a vehicle to infrastructure communication," Journal of Advanced Transportation, vol. 2020, Article ID 9139074, 2020.

[9] Z. H. Liu and C. Wang, "Design of traffic emergency response system based on internet of things and data mining in emergencies," IEEE Access, vol. 7, no. 11, pp. 3950-3962, 2019.

[10] X. J. Kong, H. R. Gao, O. Alfarraj et al., "HUAD: Hierarchical urban anomaly detection based on spatio-temporal data," IEEE Access, vol. 8, no. 26, pp. 573-582, 2020.

[11] Y. Xu, Y. L. Zhang, and M. Liu, "Multiagent based decentralized traffic light control for large urban transportation system," Mathematical Problems in Engineering, vol. 2014, Article ID 104349, 2014.

[12] R. Li, F. C. Pereira, and M. E. Ben-Akiva, "Overview of traffic incident duration analysis and prediction," European Transport Research Review, vol. 10, no. 2, p. 22, 2018.

[13] H. Cong, C. Chen, P.-S. Lin, G. Zhang, J. Milton, and Y. Zhi, "Traffic incident duration estimation based on a dual-learning Bayesian network model," Transportation Research Record: Journal of the Transportation Research Board, vol. 2672, no. 45, pp. 196-209, 2018.

[14] X. Qin and A. M. Khan, "Control strategies of traffic signal timing transition for emergency vehicle preemption," Transportation Research Part C: Emerging Technologies, vol. 25, pp. 1-17, 2012.

[15] J. So, J. Kang, S. Park, I. Park, and J. Lee, “Automated emergency vehicle control strategy based on automated driving controls," Journal of Advanced Transportation, vol. 2020, Article ID 3867921, 2020.

[16] J. Yao, K. Zhang, Y. Dai, and J. Wang, "Power function-based signal recovery transition optimization model of emergency traffic," The Journal of Supercomputing, vol. 74, no. 12, pp. 7003-7023, 2018.

[17] W. Kang, G. Xiong, Y. Lv et al., "Traffic signal coordination for emergency vehicles," in Proceedings of the 17th International IEEE Conference on Intelligent Transportation Systems (ITSC), pp. 157-161, IEEE, Qingdao, China, October 2014.

[18] H. B. Mu, L. Z. Liu, Y. B. Song, and N. Wang, "Control strategy of signal transition after emergency vehicle signal preemption," Discrete Dynamics in Nature and Society, vol. 2020, Article ID 1382415, 2020.

[19] J. Yao, K. Zhang, Y. Yang, and J. Wang, "Emergency vehicle route oriented signal coordinated control model with twolevel programming," Soft Computing, vol. 22, no. 13, pp. 4283-4294, 2018.

[20] Y.-S. Huang, Y.-S. Weng, and M. Zhou, "Design of traffic safety control systems for emergency vehicle preemption using timed Petri nets," IEEE Transactions on Intelligent Transportation Systems, vol. 16, no. 4, pp. 2113-2120, 2015.

[21] L. L. Lu and S. A. Wang, "Literature review of analytical models on emergency vehicle service: location, dispatching, routing and preemption control," in Proceedings of the 2019 IEEE Intelligent Transportation Systems Conference (ITSC), pp. 3031-3036, Auckland, New Zealand, October 2019.

[22] Z. Xie, The Control Strategy of City Traffic Occasional Congestion and Sub-Region Dynamic Partitioning Based on Graph Theory, Changsha University of Science \& Technology, Changsha, China, 2015.

[23] Y.-Q. He, Y.-L. Rong, Z.-P. Liu, and S.-P. Du, "Traffic influence degree of urban traffic emergency based on water wave principle," Journal of Transportation Systems Engineering and Information Technology, vol. 13, no. 3, 2017.
[24] A. Hofleitner, R. Herring, A. Bayen et al., Large-Scale Estimation of Arterial Traffic and Structural Analysis of Traffic Patterns from Probe Vehicles, Transportation Reasearch Board, Washington, DC, USA, 2012.

[25] E. Han, H. P. Lee, S. Park et al., "Optimal signal control algorithm for signalized intersections under a V2I communication environment," Journal of Advanced Transportation, vol. 9, 2019.

[26] R. Pueboobpaphan, D. Park, Y. Kim, and S. Choo, "Time headway distribution of probe vehicles on single and multiple lane highways," Ksce Journal of Civil Engineering, vol. 17, no. 4, pp. 824-836, 2013.

[27] A. S. Al-Ghamdi, "Analysis of time headways on urban roads: case study from Riyadh," Journal of Transportation Engineering, vol. 127, no. 4, pp. 289-294, 2001.

[28] S. M. Abtahi, M. Tamannaei, and H. Haghshenash, "Analysis and modeling time headway distribution under heavy traffic flow condition in the urban highways: case of isfahan," Transport, vol. 26, pp. 375-382, 2011.

[29] R. J. Troutbeck, "Use of Cowan's M3 headway distribution for modelling urban traffic flow," Traffic Engineering and Control, vol. 35, pp. 445-450, 1994.

[30] J. Yao, F. Li, K. Tang, and S. Jian, "Sampled trajectory datadriven method of cycle-based volume estimation for signalized intersections by hybridizing shockwave theory and probability distribution," IEEE Transactions on Intelligent Transportation Systems, vol. 21, no. 6, pp. 2615-2627, 2019.

[31] A. Z. Li, X. H. Song, Z. B. Ma et al., "Time headway distribution on downstream section of signalized intersection," Journal of Transportation Systems Engineering and Information Technology, vol. 13, 2013.

[32] Z. Li and P. Schonfeld, "Hybrid simulated annealing and genetic algorithm for optimizing arterial signal timings under oversaturated traffic conditions," Journal of Advanced Transportation, vol. 49, no. 1, pp. 153-170, 2015.

[33] Y. Xiang, S. Gubian, B. Suomela et al., "Generalized simulated annealing for global optimization: the GenSA package," $R$ Journal, vol. 5, no. 1, 2013.

[34] H. M. Amer, H. Al-Kashoash, M. Hawes, M. Chaqfeh, A. Kemp, and L. Mihaylova, "Centralized simulated annealing for alleviating vehicular congestion in smart cities," Technological Forecasting and Social Change, vol. 142, pp. 235-248, 2019.

[35] Y. Cai and H. Wang, "Migration strategy for mobile agent based on complex networks theory and genetic algorithm," Computer Engineering and Applications, vol. 41, no. 1, pp. 42-44, 2010.

[36] B. Rabbouch, F. Saadaoui, and R. Mraihi, "Empirical-type simulated annealing for solving the capacitated vehicle routing problem," Journal of Experimental \& Theoretical Artificial Intelligence, vol. 32, no. 3, pp. 437-452.

[37] Z. Xiang, W. Da, L. Chong, and R. Ji, "Analysis on Capacity of Reformed Signalized Plane intersection," in Proceedings of 2nd International Conference on Remote Sensing, Environment and Transportation Engineering, IEEE, Nanjing, China, June 2012. 